\title{
Eficacia de clúster of cuádruple differentiation (CD4) en pronóstico de pacientes con HIV.
}

\author{
Effectiveness of cluster of cuadrupe differentiation (CD4) in forecast of patients \\ with HIV.
}

Laura Judith Zúñiga Fariño. ${ }^{1}$, Lorena Paola Ramírez Morán. ${ }^{2}$, Pedro José Orellana Fariño. ${ }^{3}$ \& Nancy Corina Sánchez Coronel. ${ }^{4}$

Recibido: 11-02-2020 / Revisado: 18-03-2020 /Aceptado: 04-04-2020/ Publicado: 08-05-2020

\begin{abstract}
.
DOI: https://doi.org/10.33262/concienciadigital.v3i2.2.1242

Introduction. The human immunodeficiency virus (HIV) is one that destroys certain cells of the immune system, altering or canceling its function. A CD4 count below 200 cells / mm3 indicates serious damage to the immune system, it is a sign of AIDS in people infected with HIV, considered a public health problem, both because of the impressive morbidity and mortality figures. Objective. To determine the efficacy of Cluster of quadrupe differentiation (CD4) in prognosis of patients with HIV from Teodoro Maldonado Carbo Hospital of the year 2018. Methodology. The research design was quantitative, retrospective, descriptive and transversal. The population was 543 cases and the sample of 100 patients taken from the medical records of the AS400 system of 2018, a data collection sheet was made that consisted of 13 items, was validated by judgments of specialists and health experts qualifying validity, relevance and coherence. Results. Among the most frequent results, 57\% met the prognosis in the effectiveness of CD4, male (81\%), 25 to 35 years old (30\%), Mestizos (81\%), from the province of Guayas (85\%), active type affiliation (71\%), presented complications (73\%), tuberculosis (34\%), the initial value of CD4 at 3 months was in patients between 200 and 500 cells / mm3 with $(51 \%)$, value at 6 months was greater than 500 cells / mm3 with $(52 \%)$. Conclusion. It was concluded that more than half have CD4 efficacy, however many patients presented complications during treatment.
\end{abstract}

Keywords: Efficacy, HIV, Antiretroviral treatments, Complications, Patients.

\section{Resumen}

Introducción. El virus de la inmunodeficiencia humana (HIV) es aquel que destruye determinadas células del sistema inmunitario, alterando o anulando su función. Un conteo de CD4 por debajo de 200 células/mm3 indica daño serio al sistema inmune, es una señal del SIDA en personas infectadas con HIV, considerándose un problema de salud pública, tanto por

\footnotetext{
${ }^{1}$ Universidad Estatal de Milagro, Milagro Ecuador.lzunigaf@unemi.edu.ec

${ }^{2}$ Universidad Estatal de Milagro Facultad Ciencias de la Salud, Milagro Ecuador, lramirezm2@unemi.edu.ec

${ }^{3}$ Profesional en libre ejercicio Médico General, Milagro Ecuador, dr_pedroski@gmail.com

${ }^{4}$ Universidad Estatal de Milagro Facultad Ciencias de la Salud. Milagro Ecuador, dranancysanchez@hotmail.com
} 
las impresionantes cifras de morbilidad y mortalidad. Objetivo. Determinar la eficacia de Clúster of cuadrupe differentiation (CD4) en pronóstico de paciente con HIV del Hospital Teodoro Maldonado Carbo del año 2018. Metodología. El diseño de investigación fue cuantitativa, de tipo retrospectiva, descriptiva y transversal. La población fue de 543 casos y la muestra de 100 pacientes tomados de las historias clínicas del sistema AS400 del 2018, se realizó una ficha de recolección de datos que constó con 13 ítems, fue validado por juicios de especialistas y expertos en salud calificando validez, pertinencia y coherencia. Resultados. Entre los resultados con mayor frecuencia el 57\% cumplieron con el pronóstico en la eficacia del CD4, de sexo masculino (81\%), de 25 a 35 años de edad (30\%), Mestizos (81\%), de la provincia del Guayas (85\%), afiliación tipo activo (71\%), presentaron complicaciones $(73 \%)$, tuberculosis (34\%), el valor inicial del CD4 a 3 meses fue en pacientes entre 200 y 500 células $/ \mathrm{mm} 3$ con un (51\%), valor a los 6 meses fue mayor de 500 células $/ \mathrm{mm} 3$ con un $(52 \%)$. Conclusión. Se concluyó que más de la mitad tienen eficacia del CD4, sin embargo muchos pacientes presentaron complicaciones durante el tratamiento.

Palabras claves: Eficacia, VIH, Tratamientos antirretrovirales, Complicaciones, Pacientes.

\section{Introducción}

El HIV/sida, desconocido 35 años atrás, se ha constituido en un problema de salud mundial de una magnitud sin precedentes, considerándose que aproximadamente 25 millones de personas han fallecido en todo el mundo a causa del VIH (ONUSIDA, 2008).

En el año 2016, luego de 35 años del diagnóstico del primer caso, 36.9 millones de personas viven con HIV y 17 millones de ellas reciben terapia antirretroviral. Actualmente los antirretrovirales (ARV) han mejorado sustancialmente el pronóstico de los pacientes infectados por el virus de la inmunodeficiencia humana (HIV) a diferencia del mal pronóstico presente para la mayoría de los pacientes al inicio de la epidemia. (Chow, y otros, 2015). Esto se debe a que los ARV suprimen la replicación del VIH, lo que lleva a una disminución plasmática del ARN del virus, a un aumento de los recuentos de células T Clúster of cuadrupe differentiation (CD4), lo que se asocia a una reducción de la morbilidad y la mortalidad. (Costagliola, Lacombe, Ghosn, \& et al, 2014).

Una de cada diez personas con diagnóstico de VIH requieren de asistencia de urgencia debida a su avanzada enfermedad, las cuales cuentan con unos recuentos bajos de CD4 y por ende un riesgo alto en que la enfermedad resulte grave o mortal.

Dado que la terapia antirretroviral (TARV) modifican la morbilidad y mortalidad en los pacientes infectados por éste virus, es de gran interés identificar los predictores del resultado terapéutico (Buzon, y otros, 2014). Al respecto, los niveles de células T CD4 al inicio de la terapia antirretroviral son el más fuerte predictor de mortalidad a corto plazo. Sin embargo éste mismo recuento se vuelve menos pronóstico cuando se evalúan los resultados a los 6 meses (McNairy \& El-Sadr, 2014). A pesar de que la evidencia disponible hasta el momento señala que la carga de ARN viral a los 6 meses y los cambios en el recuento de células T CD4 a los 6 y 36 meses después del inicio de la TARV son de predictores de SIDA en pacientes con 3 años o más de supervivencia. (Lanoy, May, Mocroft, \& et al., 2009) Por este motivo, hasta la fecha, el pronóstico a corto plazo se ha extrapolado para predecir el éxito del tratamiento a largo plazo (May, y otros, 2014) (Guerrero Lara, 2016). También se conoce que los pacientes que inician una TARV con recuentos bajos de CD4 tienen una alta mortalidad, hasta 15 años después del inicio de la terapia antirretroviral. 
A fines del 2018 había alrededor de 37,9 millones de personas con el VIH, el cual continúa siendo uno de los mayores problemas para la salud pública mundial (Organización Mundial de Salud, 2019).

En Europa occidental, América del Norte y Australia, grandes colaboraciones de cohortes han podido estimar el riesgo específico de recuento de células CD4 a corto plazo de SIDA o muerte en adultos infectados con virus de inmunodeficiencia humana (HIV) no tratados con altos recuentos de células CD4. En el África subsahariana, estas estimaciones específicas de conteo de células CD4 son escasas. (Noda Albelo, Vidal Tallet, Pérez Lastre, \& Cañete Villafranca, 2014) Debido a lo incompleto de los datos existentes, no está claro si las pautas de tratamiento de la infección por HIV deben ser específicas de cada región. Existe una amplia evidencia que muestra que algunas enfermedades infecciosas, como la tuberculosis, son más frecuentes en el África subsahariana que en Europa o en los Estados Unidos, tanto en la población general y en la población infectada por el VIH. Sin embargo, debido a que las tasas específicas de recuento de células CD4 de estas enfermedades no se han estimado en todo el espectro de recuentos de células CD4 antes de la era ART, la cuestión de si los pacientes con recuentos de células CD4 similares deben tener diferentes pautas, dependiendo de la región en la que ellos viven, no ha sido claramente abordado. Esto es particularmente cierto cuando se trata de la cuestión crucial de cuándo comenzar ART.

De estos últimos estudios, algunos se realizaron en la década de 1990, cuando los recuentos de células CD4 no estaban disponibles, y la mayoría de los otros describieron la morbilidad en base a los recuentos de células CD4 basales y no de seguimiento. (Boza Cordero, 2016)

Hay que ser conscientes que los niveles de estas infecciones crecen a un ritmo acelerado con niveles altos a nivel mundial, convirtiéndose un problema de salud pública, las cifras de nuevos casos siguen siendo alarmantes. Es el HIV que se encuentra en gran cantidad para su transmisión en la sangre, secreciones vaginales, semen, líquido amniótico además en la leche materna de la madre infectada. Por lo tanto, se reconoce tres vías de transmisión: la vía sexual, perinatal o vertical y sanguínea. Son los medios de formas directas donde se transmite el virus. Tiene como célula blanco de infección a aquellas que muestran receptores de CD4, cómo los linfocitos T del linaje CD4, y tambien varios macrófagos. Lo que genera daños en la célula blanco y como consecuencia la escasez del sistema inmune dejando así delicado al organismo de infecciones debido a los agentes infecciosos oportunistas y a la vez el aumento de las células cancerígenas normalmente controladas por el sistema inmune.

La problemática existente en los pacientes con HIV es una pandemia espasmódica ya que en cada año el número de paciente va en aumento, tanto a nivel mundial, nacional y dentro del Hospital Teodoro Maldonado Carbo el cual se encuentra ubicado en la ciudad de GuayaquilEcuador. Los laboratorios de la Institución se los ha implementado con tecnología que otras instituciones no la logran tener, por los costos elevados. Para lo cual se puede estudiar los valores de CD4 de los pacientes que ingresan con diagnostico reciente de HIV.

En la actualidad se recibe aproximadamente 550 pacientes nuevos cada año con diagnóstico de HIV. La institución cuenta con un laboratorio del cual los pacientes acuden a exámenes del CD4, la máquina que realiza estos exámenes aparte del valor por la cual fue adquirida también en ocasiones requiere mantenimiento generando así gastos intra-hospitalario, Además el aumento de días de estancia hospitalaria provoca un incremento en los costos hospitalarios por paciente y un mayor gasto de bolsillo para el familiar, convirtiéndose así en un problema de salud pública por los altos desembolsos económico que afecta al estado, a la familia y las instituciones hospitalaria. A muchos de los pacientes se les ha administrado antiretrovirales 
presentado eventos adversos prolongando estancia hospitalaria dando complicaciones, aumentando la incapacidad del paciente y/o la muerte, sin contar las complicaciones propias de la enfermedad del VIH como la tuberculosis, citomegalovirus, toxoplasmosis, sarcoma de kaposi, linfoma, cryptococcus, etc. Por todo esto y mucho más debe ser considerado de suma importancia por las instituciones públicas.

Por lo tanto, el propósito u objetivo de este presente trabajo investigativo es de determinar la eficacia de Clúster of cuadrupe differentiation (CD4) en pronóstico de paciente con HIV, el cual consiste en identificar las características socio-demográficas y los pronósticos de CD4 de los pacientes con HIV del Hospital Teodoro Maldonado Carbo, las complicaciones que presentaron los pacientes con HIV sometidos a pruebas del CD4 y analizar los valores de CD4 y el tipo de resultado terapéutico obtenido con el TARV.

\section{Método}

El diseño y tipo de la investigación fue cuantitativa, de tipo retrospectiva, transversal y descriptiva, de tipo no experimental, el estudio estuvo enfocado a pacientes que ingresaron con diagnóstico de HIV, que acudieron al Laboratorio inmunológico del HTMC de la ciudad de Guayaquil., en el año 2018, la población fue de 543 pacientes, y el tamaño de muestra fue de 100 pacientes el cual se aplicó una formula estadística de la población finita con un margen de error del $0.05 \%$, el proceso de selección fue aleatorio, los datos fueron tomados directamente de las historias clínicas del Sistema AS400 el cual se contó con el apoyo del director del Hospital para poder tomar los datos de los pacientes para el trabajo a cabo. Se utilizó como instrumento una ficha de recolección de datos validada por juicio de expertos en salud pública, el cual constó con 13 ítems de manera organizada en relación a cada objetivo del estudio presente, siendo validados por juicios de expertos en salud pública calificando validez un $98 \%$, pertinencia $96 \%$ y confiabilidad un $98 \%$.

El instrumento constó con los siguientes ítems; eficacia de Clúster of cuadrupedifferentiation (CD4) en pronóstico de paciente con HIV, el cual el primer ítems índico sobre la eficacia del CD4; (Cumple con el pronóstico - No cumple con el pronóstico), sexo (Masculino Femenino), el grupo etario (25 a 35 Años - 36 a 45 Años - 46 a 55 Años - 56 a 65 Años Más de 66 Años), Raza (Mestizo - Indígena - Blanca - Negro - montubio), Procedencia (Guayas - Esmeraldas - Los Ríos - Pichincha - Manabí - Imbabura - El Oro), Afiliación (Activo - Seguro Campesino - Voluntario - Jubilado - Extensión de cónyuge - montepío), Pronóstico de los pacientes que realizaron el CD4 (Presentaron Complicaciones - No presentaron Complicaciones), complicación presentadas en los pacientes (Tuberculosis Toxoplasmosis - Citomegalovirus - Sarcoma de Kaposi - Linfoma - Cryptococcus - Otros Ninguna) y Adherencia ( $\mathrm{Si}-\mathrm{No}$ ), valores iniciales estableciendo un rango de cuyos valores, (CD4+ > 500 células/mm3 - CD4 + entre 200 y 500 células/mm3 - CD4+< 200 células/mm3) las mismas alterativas de los rangos se utilizó para conocer los valores durante 3 y 6 meses.

\section{Resultados}

Los resultados de los 100 casos, se verificó la Eficacia del CD4 que tuvieron los pacientes con HIV en el año 2018 en el HTMC, 57 pacientes si cumplieron con el pronóstico el cual corresponden a un 57\%, mientras que 43 pacientes no cumplieron con el pronóstico equivalente a un $43 \%$. Por lo tanto, se pudo verificar que más de la mitad de la muestra estudiada si cumplen el pronóstico, sin embargo, existe una gran parte en que los pacientes no cumplen con los pronósticos establecidos, como se refleja en la tabla $\mathrm{N}^{\circ} 1$. 
Tabla 1. Eficacia del CD4 en los pacientes con HIV

\begin{tabular}{lll}
\hline Eficacia del CD4 & Frecuencia & Porcentaje \\
\hline Cumple con el pronóstico & 57 & $57,00 \%$ \\
No Cumple con el pronóstico & 43 & $43,00 \%$ \\
Total & 100 & $100,00 \%$ \\
\hline
\end{tabular}

Elaborado: por la Dra. Laura Judith Zúñiga Fariño

Fue de suma importancia saber las características sociodemográficas de los pacientes con HIV de los cuales existieron $81 \%$ en casos de sexo masculino, mientras que el sexo femenino fue de $19 \%$. El rango de edad que en ese entonces tenían los pacientes el cual se determinó que de 25 a 35 años se presentaron 30\%, de 36 a 45 años $28 \%$, de 46 a 55 Años un 22\%, de 56 a 65 Años $16 \%$, mientras que más de 66 años fue un $4 \%$. La raza de los pacientes el $81 \%$ era mestiza, $5 \%$ fue Indígena, $5 \%$ blanca, $5 \%$ negra y el $4 \%$ montubio. La procedencia de algunas de las provincias del Ecuador se pudo identificar que el $84 \%$ eran del Guayas provincia donde queda el hospital, Esmeraldas un 4\%, Los Ríos 3\%, Pichincha 3\%, Manabí 3\%, Imbabura 1\% y en el Oro $2 \%$. El tipo de afiliación en de los pacientes el $71 \%$ fue activo, el $9 \%$ por seguro campesino, el $8 \%$ voluntario, el $7 \%$ Jubilado, el $2 \%$ por extensión de cónyuge y el $3 \%$ montepío, cuyos resultados se reflejan en la tabla $\mathrm{N}^{\circ} 2$.

Tabla 2. Características sociodemográficas de los pacientes con HIV

\begin{tabular}{|c|c|c|c|}
\hline Sexo & & & \\
\hline & & Frecuencia & Porcentaje \\
\hline & Masculino & 81 & $81,00 \%$ \\
\hline & Femenino & 19 & $19,00 \%$ \\
\hline & Total & 100 & $100 \%$ \\
\hline \multicolumn{4}{|c|}{ Grupo Etario } \\
\hline & 25 - 35 Años & 30 & $30,00 \%$ \\
\hline & $36-45$ Años & 28 & $28,00 \%$ \\
\hline & 46 - 55 Años & 22 & $22,00 \%$ \\
\hline & $56-65$ Años & 16 & $16,00 \%$ \\
\hline & $\begin{array}{l}\text { Más de } 66 \\
\text { Años }\end{array}$ & 4 & $4,00 \%$ \\
\hline & Total & 100 & $100 \%$ \\
\hline \multicolumn{4}{|l|}{ Raza } \\
\hline & Mestiza & 81 & $81,00 \%$ \\
\hline & Indígena & 5 & $5,00 \%$ \\
\hline & Blanca & 5 & $5,00 \%$ \\
\hline & Negra & 5 & $5,00 \%$ \\
\hline & Montubia & 4 & $4,00 \%$ \\
\hline & Total & 100 & $100 \%$ \\
\hline \multicolumn{4}{|c|}{ Lugar de Procedencia } \\
\hline & Guayas & 84 & $84,00 \%$ \\
\hline & Esmeraldas & 4 & $4,00 \%$ \\
\hline & Los Ríos & 3 & $3,00 \%$ \\
\hline & Pichincha & 3 & $3,00 \%$ \\
\hline
\end{tabular}


Vol. 3, N².2, p. 6-18, junio, 2020

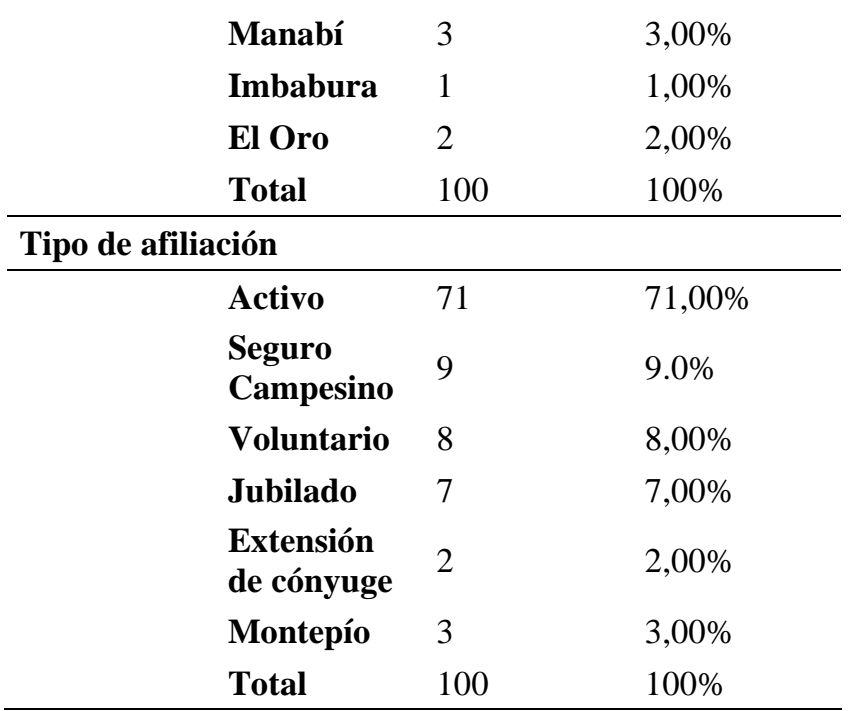

Elaborado: por la Dra. Laura Judith Zúñiga Fariño

El pronóstico de pacientes al realizar la prueba del CD4 por primera vez, el $73 \%$ tuvieron complicaciones, mientras que el $27 \%$ de los pacientes no presentaron ninguna complicación. Entre las complicaciones que presentaron los pacientes con HIV, el 34\% presentaron tuberculosis, el 13 de ellos con toxoplasmosis, el 8\% de los pacientes con Linfoma, el $7 \%$ con citomegalovirus, el 5\% con sarcoma de kaposi, el 4\% con Cryptococcus, el $20 \%$ en otras complicaciones. Por lo tanto, se pudo determinar que la complicación en pacientes con HIV más frecuente fue debido a la tuberculosis seguido de la toxoplasmosis, tal como se observa en la tabla $\mathrm{N}^{\circ} 3$.

Tabla 3. Complicaciones que presentaron los pacientes.

\begin{tabular}{|c|c|c|c|c|c|}
\hline & & & \multicolumn{2}{|c|}{$\begin{array}{c}\text { Pronóstico de pacientes al } \\
\text { realizar prueba CD4 }\end{array}$} & \multirow[b]{2}{*}{ Total } \\
\hline & & & $\begin{array}{l}\text { Presentaron } \\
\text { Complicaciones }\end{array}$ & $\begin{array}{c}\text { No } \\
\text { presentaron } \\
\text { Complicación } \\
\end{array}$ & \\
\hline \multirow{15}{*}{ Complicaciones } & \multirow{2}{*}{ Tuberculosis } & Recuento & 34 & 0 & 34 \\
\hline & & $\%$ del total & $34,00 \%$ & $0,00 \%$ & $34,00 \%$ \\
\hline & \multirow{2}{*}{ Toxoplasmosis } & Recuento & 13 & 0 & 13 \\
\hline & & $\%$ del total & $13,00 \%$ & $0,00 \%$ & $13,00 \%$ \\
\hline & \multirow{2}{*}{ Citomegalovirus } & Recuento & 7 & 0 & 7 \\
\hline & & $\%$ del total & $7,00 \%$ & $0,00 \%$ & $7,00 \%$ \\
\hline & \multirow[b]{2}{*}{ Sarcoma de Kaposi } & Recuento & 5 & 0 & 5 \\
\hline & & $\%$ del total & $5,00 \%$ & $0,00 \%$ & $5,00 \%$ \\
\hline & \multirow{2}{*}{ Linfoma } & Recuento & 8 & 0 & 8 \\
\hline & & $\%$ del total & $8,00 \%$ & $0,00 \%$ & $8,00 \%$ \\
\hline & \multirow{2}{*}{ Cryptococcus } & Recuento & 4 & 0 & 4 \\
\hline & & $\%$ del total & $4,00 \%$ & $0,00 \%$ & $4,00 \%$ \\
\hline & \multirow{2}{*}{ Otros } & Recuento & 2 & 0 & 2 \\
\hline & & $\%$ del total & $2,00 \%$ & $0,00 \%$ & $2,00 \%$ \\
\hline & Ninguna & Recuento & 0 & 27 & 27 \\
\hline
\end{tabular}


Vol. 3, N².2, p. 6-18, junio, 2020

\begin{tabular}{ccccc}
\hline & $\%$ del total & $0,00 \%$ & $27,00 \%$ & $27,00 \%$ \\
\hline \multirow{2}{*}{ Total } & Recuento & 73 & 27 & 100 \\
& $\%$ del total & $73,00 \%$ & $27,00 \%$ & $100,00 \%$ \\
\hline
\end{tabular}

Elaborado: por la Dra. Laura Judith Zúñiga Fariño

La adherencia fue de suma importancia considerar en este estudio ya que el paciente recibe instrucciones antes de iniciar la terapia y esté totalmente dispuesto a realizar el tratamiento, donde el $83 \%$ si tuvieron adherencia, mientras que el 17\% no tuvieron adherencia. Tal como se muestra en la tabla $\mathrm{N}^{\circ} 4$.

Tabla 4. Adherencia

\begin{tabular}{ccc}
\hline Adherencia & Frecuencia & Porcentaje \\
\hline Si & 83 & $83,00 \%$ \\
No & 17 & $17,00 \%$ \\
Total & 100 & $100,00 \%$ \\
\hline
\end{tabular}

Elaborado: por la Dra. Laura Judith Zúñiga Fariño

En el estudio se dio a conocer los valores del CD4 desde el inicio hasta los 6 meses, en los pacientes sometidos a exámenes del CD4 se pudo determinar que el $51 \%$ de los pacientes tuvieron entre 200 y 500 células $/ \mathrm{mm} 3$, el $29 \%$ fueron mayores a 500 células/mm3, mientras que el $20 \%$ hubieron casos menores de 200 células/mm3. Por lo tanto, indica que la gran mayoría de los pacientes presentaron valores iníciales entre 200 a 500 células/mm3 es decir que el sistema inmunitario está gravemente debilitado y se hace necesario el TARV. Con respecto a los valores del CD4 durante 3 meses en los pacientes se pudo verificar que se mantiene el mismo porcentaje en los valores del 200 a 500 células/mm3 con un 51\%, pero cabe recalcar que aumento una pequeña parte de la muestra de valores mayores a 500 células $/ \mathrm{mm} 3$ con un 33\%, mientras que redujo el índice de pacientes con valores menores a 200 células/mm3. Los valores del CD4 durante 6 meses, se pudo evidenciar que existió un gran incremento del $52 \%$ de los pacientes que tuvieron más de 500 células $/ \mathrm{mm} 3$ por lo que se comprueba que el paciente tuvo buena adherencia, el cual solo quedo un 33\% de pacientes con valores entre 200 a 500 células/mm3 y el 15\% de pacientes con valores menores a 200 células/mm3. Los resultados de recuperación con el TARV que reflejo la historia clínica de los pacientes el $52 \%$ si recupera, sin embargo el $48 \%$ no, como se observa en la tabla $\mathrm{N}^{\circ} 5$.

Tabla 5. Valores del CD4 y Recuperación con el TARV de los pacientes con HIV.

\begin{tabular}{lll}
\hline Valores Iniciales & Frecuencia & Porcentaje \\
\cline { 2 - 3 } CD4+ $>\mathbf{5 0 0}$ células/mm3 & 29 & $29,0 \%$ \\
CD4+ entre 200 a 500 células/mm3 & 51 & $51,0 \%$ \\
CD4+ $<200$ células/mm3 & 20 & $20,0 \%$ \\
\hline Total & 100 & $100 \%$ \\
\hline Valores a los 3 meses & & \\
CD4+ $>\mathbf{5 0 0}$ células/mm3 & 33 & $33,0 \%$
\end{tabular}




\begin{tabular}{lll} 
CD4+ entre 200 a 500 células/mm3 & 51 & $51,0 \%$ \\
CD4+ < 200 células/mm3 & 16 & $16,0 \%$ \\
\hline Total & 100 & $100 \%$ \\
\hline Valores a los $\mathbf{6}$ meses & & \\
CD4+ > 500 células/mm3 & 52 & $52,0 \%$ \\
CD4+ entre 200 a 500 células/mm3 & 33 & $33,0 \%$ \\
CD4+ < 200 células/mm3 & 15 & $15,0 \%$ \\
\hline Total & 100 & $100 \%$ \\
\hline Resultados de Recuperación con el TARV & \\
Recupera & 52 & $52,0 \%$ \\
No Recupera & 48 & $48,0 \%$ \\
Total & 100 & $100 \%$ \\
\hline
\end{tabular}

Elaborado: por la Dra. Laura Judith Zúñiga Fariño

\section{Discusión}

El estudio realizado permitió conocer a los pacientes si tuvieron eficacia, entre los resultados el 57\% si cumplió con el pronóstico, mientras el 43\% no cumple con el pronóstico.

Según el estudio Susana Gabriela Cabrera Alonso en el año 2018 realizado en Uruguay con el tema "Calidad de vida relacionada a la salud en personas con VIH y factores asociados" el estudio fue transversal de muestra no probabilística de personas con VIH, mayores de 18 años, que recibiendo TARV, asistidas en el subsector público en un período de 24 meses. En donde el $86 \%$ si cumplieron con el pronóstico necesario, mientras que el $14 \%$ no lo cumplieron. (Cabrera Alonso, Arteta Dalchiele, Meré Rouco, \& Cavalleri Ferrari, 2018).

Por lo tanto, al comparar dichos resultados con el presente estudio se pudo comprobar que existe un nivel similitud ya que en ambos estudios la gran mayoría de los pacientes si cumplieron con el pronóstico.

En el presente estudio se determinó que el sexo masculino abarco un $81 \%$ de pacientes con HIV y el sexo femenino fue de $19 \%$.

Según el estudio de Juana Pacífico y César Gutiérrez en el año 2015 realizaron una investigación con el tema; "Información sobre la medicación y adherencia al tratamiento antirretroviral de gran actividad en pacientes con VIH/Sida de un Hospital de Lima, Perú" el estudio fue de corte transversal analítico. Por medio de historias clínicas se recogieron 364 datos sociodemográficos y clínicos de los pacientes, entre los resultados se pudo comprobar que el sexo masculino frecuento más en el estudio con un porcentaje del 70,3\%, mientras que el sexo femenino abarco un 29,7\% de la población del estudio. (Pacífico \& Gutiérrez, 2015)

Al comparar los resultados del presente estudio se constató que existe una gran relación con los resultados ya que en ambos estudios el sexo masculino con los que más conllevan esta enfermedad.

Otra variable sociodemográfica que fue muy importante considera fue el rango de edad entre los 25 a 35 abarco un 30\%, el $28 \%$ de 36 a 45, el 22\% de 46 a 55 Años, el $16 \%$ de 56 a 65 Años y el $4 \%$ más de 66 años. 
Según el estudio de Maryenis Rodríguez Alfaro realizado en el año 2011 con el tema; "Efectividad del tratamiento antirretroviral en pacientes con virus de inmunodeficiencia humana/sida en un hospital de Zimbabwe", fue una investigación descriptiva y prospectiva de 171 pacientes con virus de inmunodeficiencia humana/sida, que recibieron tratamiento antirretroviral desde abril hasta diciembre de 2008 en el Hospital de Bindura de la provincia de Mashonaland Central (Zimbabwe), las edad que comprendió con mayor frecuencia fue en el rango de 29 a 43 años con un 60,8\%, seguido del rango de edad de 44 a 58 años con un $24 \%$, el $11,7 \%$ de 14 a 28 años, mientras que desde los 59 a 73 años el 3,5\%. (Rodríguez Alfaro, Gross Fernández, \& Casas Gross, 2016)

A comparar ambos estudios se pudo observar que no fueron clasificados por igual el rango de edades, sin embargo, se notó que de todas formas la edad comprendida desde los 25 a 45 años es donde la enfermedad más se manifiesta. Por lo tanto, se admite que dichos resultados tienen un grado de similitud.

Se sabe que el pronóstico de los pacientes que realizaron la prueba del CD4 por primera vez el $73 \%$ tuvo complicaciones mientras que el $27 \%$ no pasaron por complicaciones.

Según el estudio de Rosa Martín Cabo, en el año 2012 el tema; "Promoción de la detección del virus de la inmunodeficiencia humana en atención primaria" realizado en la ciudad de Barcelona, España. El estudio fue de intervención no aleatorizado de un centro de salud urbano en pacientes de 18 a 65 años de edad a quienes se iba a realizar prueba del CD4, la población es de 209 en donde la gran mayoría presentaron complicaciones aproximadamente un 67,4\% lo presentaban mientras que el $32,6 \%$ no presentaban complicaciones. (Cabo, Losa García, Iglesias Franco, \& Iglesias González, 2014)

Por lo tanto, se pudo determinar que en ambos estudios existió un índice alto de pacientes que presentaron complicaciones durante la prueba del CD4.

En este estudio la adherencia en el tratamiento fue del $83 \%$, mientras que el $17 \%$ no tuvieron adherencia.

Según el estudio de Maria T. Varela Arévalo, en el año 2015 realizó una investigación con el tema "La adherencia al tratamiento para el VIH/SIDA: más allá de la toma de antirretrovirales" fue de tipo observacional transversal, con una muestra de 277 personas con VIH/Sida de nueve instituciones de salud. Se utilizó el cuestionario de adherencia al tratamiento para el VIH/Sida, en donde solo el $37 \%$ de las personas fueron adherentes al tratamiento no farmacológico, mientras que el $63 \%$ no lo fueron. (Varela Arévalo \& Hoyos Hernández, 2015)

Los resultados a cabo en comparación al estudio de Varela se pudo verificar que existió una gran diferencia, ya que en el estudio presente la gran mayoría si fueron adherentes mientras que en el estudio anterior más de la mitad de la población no fueron adherentes.

Entre las complicaciones dadas en este estudios que presentaron los pacientes con HIV, la tuberculosis abarco un 34\%, la toxoplasmosis un 13\%, el Linfoma un 8\%, el citomegalovirus un 7\%, la sarcoma de Kaposi un 5\%, el Cryptococcusun 4\%, otras complicaciones el 20\%, mientras que el $27 \%$ ninguna complicación.

El estudio de Caja Calvo, realizado en el año 2007 con el tema; "Supervivencia de las mujeres infectadas por el VIH tras la aparición del TARGA" fue de tipo retrospectivo, con una muestra de 48 pacientes en donde la complicación que más incidió en ese entonces fue la tuberculosis con un $25 \%$, la candidiasis esofágica, traqueal, bronquial o pulmonar abarco con un $12,5 \%$, el cáncer de cérvix con un $10,4 \%$, la toxoplasmosis un 8,3\%, el Linfoma con un 7,2\%, Infección 
por herpes virus diseminada un 4,2\% y la histoplasmosis diseminada con un 2\%. (Caja Calvo, Rocher Milla, Flores Cid, Soler Company, \& Peris Ribera, 2015)

Por lo tanto, se pudo comparar que en ambos estudios la tuberculosis es la complicación que más se ha presentado en los pacientes con HIV, el cual constituye una complicación oportunista muy frecuente en el seno de la infección producida por el HIV.

En el presente estudio se analizó los valores iníciales del CD4 y luego se tomaron cuyos valores durante 3 meses y 6 meses. Durante los 3 meses el $51 \%$ fueron 200 a 500 células/mm3 de los resultados de los pacientes un $33 \%$ mayor a 500 células $/ \mathrm{mm} 3$ y el $16 \%$ entre los valores menores a 200 células/mm3. Pero con los datos tomados luego de 6 meses se notó una gran diferencia ya que los resultados del CD4 reflejaron que incremento el 52\% de los pacientes con más de 500 células/mm3, el $33 \%$ entre 200 a 500 células/mm3 y el $15 \%$ menores a 200 células/mm3.

Retomando el estudio de Caja Calvo, realizado en el año 2007, realizó su investigación siguiendo los estadios inmunológico y clínico (en función de la clasificación CDC) de los pacientes en el diagnóstico de su enfermedad realizando la prueba del CD4, el cual el estudio fue enfocado en diferentes rangos de años el cual el primer rango indico que el 43,7\% tuvieron mayor de 500 células/mm3, el 39,6\% entre el rango de 250 a 500 células $/ \mathrm{mm} 3$, mientras que el 16,7\% menores de 250 células/mm3. Sin embargo, en el segundo rango de años los valores tomaron otras cantidades, el 44,4\%, estuvo entre los 250 a 500 células $/ \mathrm{mm} 3$, el 29,6\% menores a 250 células $/ \mathrm{mm} 3$, mientras que el $26 \%$ fueron mayores a 500 células/mm3. Al comparar ambos años se pudo verificar que los pacientes que se encontraban con más de 500 células $/ \mathrm{mm} 3$ en el siguiente rango de edad redujeron casi a la mitad. (Caja Calvo, Rocher Milla, Flores Cid, Soler Company, \& Peris Ribera, 2015).

Por lo tanto, se pudo verificar en el presente estudio en relación al estudio de Caja que los valores iníciales en comparación a los valores posteriores surgieron ciertos cambios, en el estudio de Caja se reflejó que los valores del CD4 mayores a 500 células $/ \mathrm{mm} 3$ reducieron casi a la mitad de la población que se asumía que estaban bien en su primer prueba. Sin embargo, en el presente estudio se observe que más de la mitad de los pacientes tenían valores de 200 a 500 células/mm3 luego de 6 meses se les realizó otra prueba del CD4 donde resultó que más de la mitad tenían más de 500 células/mm3 es decir que ciertos pacientes si tuvieron Buenos resultados.

\section{Conclusión}

- Se determinó en el presente estudio que más de la mitad de la muestra estudiada de los pacientes con HIV del Hospital Teodoro Maldonado Carbo del año 2018, si presentaron elevación de los niveles de CD4. Sin embargo, se debe considerar que de todas formas existió una gran transcendencia en pacientes que no tuvieron eficacia de los tratamientos pues se los debe mantenerlo de por vida, muchos de los pacientes dejan su medicina, el cual es considerado un problema de salud pública debido al alto índice de características negativas por parte de la familia, las condiciones económicas bajas, generando mayor inversión tanto para la población, inversión hospitalaria y gubernamental.

- Entre las características sociodemográficas más relevantes fueron los de sexo masculino en un rango de edad de 25 a 35 Años, donde la gran mayoría presentaron descenso en los niveles del examen del CD4, debido al no seguir los tratamientos antirretrovirales, el cual se ha convertido en un problema de salud pública y un serio problema social por el contexto discriminatorio que existe hacia el contagiado, además 
están en la edad productiva, pero muchos de los pacientes deben ser hospitalizados dejando de ser personas activas laboralmente mientras su cuadro clínico evolucione, favorablemente o no.

- Las complicaciones que con mayor frecuencia incidió en los pacientes con HIV sometidos a las pruebas de laboratorio del CD4, fue la Tuberculosis el cual, es una de las causas más importantes de enfermedad y muertes en muchos países y un importante problema de salud pública ya que es una de las 10 principales causas de mortalidad en el mundo, los grandes costos que conlleva las complicaciones y la enfermedad perse.

- Se analizó los valores de CD4 de los pacientes obtenidos de los resultados terapéuticos con el TARV, tanto valor inicial, 3 y 6 meses, entre los valores iníciales y de 3 meses indicaron tener un margen de similitud del CD4 de 200 a 500 células/mm3, sin embargo, luego de 6 meses reflejó que más de la mitad reflejaron un incremento de más de 500 células $/ \mathrm{mm} 3$, es decir que dichos pacientes mejoraron. El cual se ha establecido la importancia del conteo de CD4 el cual se utilizar para vigilar la salud del sistema inmunitario en personas infectadas con el VIH a pesar que se saber que no hay medicamentos que protegen el sistema inmunitario pero pueden prevenir el Sida siguiendo los tratamientos.

\section{Referencias Bibliográficas}

Boza Cordero, R. (2016). Orígenes del VIH/SIDA. Revista Clínica de la Escuela de Medicina UCR - HSJD, 6(4), 48-60. Obtenido de https://www.medigraphic.com/pdfs/revcliescmed/ucr-2016/ucr164g.pdf

Buzon, M., Martin-Gayo, E., Pereyra, F., Ouyang, Z., Sun, H., Li, J., \& Lichterfeld, M. (2014). Long-term antiretroviral treatment initiated at primary HIV-1 infection affects the size, composition, and decay kinetics of the reservoir of HIV-1-infected CD4 T cells. J Virol, 88(17), 10056-10065. Obtenido de https://www.ncbi.nlm.nih.gov/pubmed/24965451

Cabo, R. M., Losa García, J. E., Iglesias Franco, H., \& Iglesias González. (2014). Promoción de la detección del virus de la inmunodeficiencia humana en atención primaria. Gaceta Sanitaria, 26(2), 116-122. Obtenido de http://scielo.isciii.es/pdf/gs/v26n2/original2.pdf

Cabrera Alonso, S. G., Arteta Dalchiele, Z., Meré Rouco, J. J., \& Cavalleri Ferrari, F. (2018). Calidad de vida relacionada a la salud en personas con VIH y factores asociados. Revista Médica Uruguay, 34(1), 7-19. Obtenido de scielo.edu.uy/pdf/rmu/v34n1/16880390-rmu-34-01-00003.pdf

Caja Calvo, A., Rocher Milla, J., Flores Cid, E., Soler Company, J. E., \& Peris Ribera. (2015). Supervivencia de las mujeres infectadas por el VIH tras la aparición del TARGA. Anales de Medicina Interna, 24(10). Obtenido de http://scielo.isciii.es/pdf/ami/v24n10/original4.pdf

Chow, W., Lim, S., Ong, L., Yong, Y., Takebe, Y., Kamarulzaman, A., \& Tee, K. (2015). Impact of HIV-1 Subtype on the Time to CD4+ T-Cell Recovery in Combination Antiretroviral Therapy (cART)-Experienced Patients. PLoS ONE, 10(9), 0137281.

Costagliola, D., Lacombe, J., Ghosn, J., \& et al. (2014). CD4+ cell count recovery in naive patients initiating ART, who achieved and maintained plasma HIV-RNA suppression. J Int AIDS Soc, 17(4 suppl 3), 19481. 
Guerrero Lara, A. J. (2016). Trends over calendar time in antiretroviral treatment success and failure in HIV clinic populations. HIV Med, 11, 432-8.

Lanoy, E., May, M., Mocroft, A., \& et al. (2009). Prognosis of patients treated with ART from 36 months after initiation, according to current and previous CD4 cell count and plasma HIV-1 RNA measurements. AIDS, 23, 2199-208.

May, M., Ingle, S., Costagliola, D., Justice, A., de Wolf, F., \& Cavassini, M. (2014). Cohort profile: Antiretroviral Therapy Cohort Collaboration (ART-CC). Int J Epidemiol, 43, 691-702.

McNairy, M., \& El-Sadr, W. (2014). Antiretroviral therapy for the prevention of HIV transmission: what will it take? Clin. Infect. Dis., 1003-1011.

Noda Albelo, A. L., Vidal Tallet, L. A., Pérez Lastre, J. E., \& Cañete Villafranca, R. (2014). Interpretación clínica del conteo de linfocitos T CD4 positivos en la infección por VIH. Revista Cubana de Medicina, 52(2), 118-127. Obtenido de http://scielo.sld.cu/pdf/med/v52n2/med05213.pdf

ONUSIDA. (2008). Informe sobre la epidemia Mundial del Sida. Programa Conjunto de las Naciones Unidas sobre el VIH/sida. México: Catalogación por la Biblioteca de la OMS. Obtenido de http://data.unaids.org/pub/globalreport/2008/jc1510_2008globalreport_es.pdf

Organización Mundial de Salud. (4 de Septiembre de 2019). VIH/Sida. Obtenido de Estrategia mundial del sector de la salud contra el VIH, 2016-2021: https://www.who.int/es/newsroom/fact-sheets/detail/hiv-aids

Pacífico, J., \& Gutiérrez, C. (2015). Información sobre la medicación y adherencia al tratamiento antirretroviral de gran actividad en pacientes con VIH/Sida de un Hospital de Lima, Perú. Rev. Peru Med Exp Salud Pública, 32(1), 66-72. Obtenido de http://www.scielo.org.pe/pdf/rins/v32n1/a10v32n1.pdf

Rodríguez Alfaro, M., Gross Fernández, C., \& Casas Gross, S. (2016). Efectividad del tratamiento antirretroviral en pacientes con virus de inmunodeficiencia humana/sida en un hospital de Zimbabwe. Revista MEDISAN, 15(4), 433-441. Obtenido de http://scielo.sld.cu/pdf/san/v15n4/san05411.pdf

Varela Arévalo, M. T., \& Hoyos Hernández, P. A. (2015). La adherencia al tratamiento para el VIH/SIDA: más allá de la toma de antirretrovirales. Revista de Salud Pública, 17(4), 528-540. Obtenido de https://www.scielosp.org/pdf/rsap/2015.v17n4/528-540/es 


\section{PARA CITAR EL ARTÍCULO INDEXADO.}

Zúñiga Fariño, L. J., Ramírez Morán, L. P., Orellana Fariño, P. J., \& Sánchez Coronel, N. C. (2020). Eficacia de clúster of cuádruple differentiation (CD4) en pronóstico de pacientes con HIV. ConcienciaDigital, 3(2.2), 6-18. https://doi.org/10.33262/concienciadigital.v3i2.2.1242

\section{Liencia}

El artículo que se publica es de exclusiva responsabilidad de los autores y no necesariamente reflejan el pensamiento de la Revista Conciencia Digital.

El artículo queda en propiedad de la revista y, por tanto, su publicación parcial y/o total en otro medio tiene que ser autorizado por el director de la Revista Conciencia Digital.

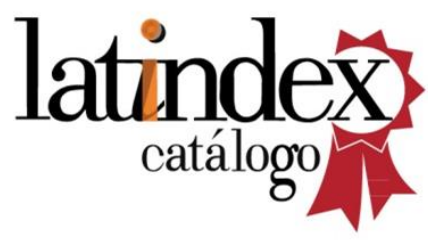

